\title{
Synthesis of Cell-Adhesive Anisotropic Multifunctional Particles by Stop Flow Lithography and Streptavidin-Biotin Interactions
}

\author{
Ki Wan Bong ${ }^{\dagger, \ddagger, \perp}$, Jae Jung Kim ${ }^{\S, \perp}$, Hansang $\mathrm{Cho}^{\dagger}$, Eugene Lim ${ }^{\dagger}$, Patrick S. Doyle ${ }^{\S,}{ }^{*}$, and \\ Daniel Irimia ${ }^{*} \dagger$ \\ ${ }^{\dagger}$ Center for Engineering in Medicine and Surgical Services, Massachusetts General Hospital, \\ Harvard Medical School, Charlestown, Massachusetts 02129, United States \\ ‡Department of Chemical and Biological Engineering, Korea University, Anam-dong, Seongbuk- \\ gu, Seoul 136-713, Korea
}

$\S$ Department of Chemical Engineering, Massachusetts Institute of Technology, Cambridge, Massachusetts 02139, United States

\begin{abstract}
Cell-adhesive particles are of significant interest in biotechnology, the bioengineering of complex tissues, and biomedical research. Their applications range from platforms to increase the efficiency of anchorage-dependent cell culture to building blocks to loading cells in heterogeneous structures to clonal-population growth monitoring to cell sorting. Although useful, currently available celladhesive particles can accommodate only homogeneous cell culture. Here, we report the design of anisotropic hydrogel microparticles with tunable cell-adhesive regions as first step toward micropatterned cell cultures on particles. We employed stop flow lithography (SFL), the coupling reaction between amine and $N$-hydroxysuccinimide (NHS) and streptavidin-biotin chemistry to adjust the localization of conjugated collagen and poly-L-lysine on the surface of microscale particles. Using the new particles, we demonstrate the attachment and formation of tight junctions between brain endothelial cells. We also demonstrate the geometric patterning of breast cancer cells on particles with heterogeneous collagen coatings. This new approach avoids the exposure of cells to potentially toxic photoinitiators and ultraviolet light and decouples in time the microparticle synthesis and the cell culture steps to take advantage of the most recent advances in cell patterning available for traditional culture substrates.
\end{abstract}

\section{Graphical abstract}

\footnotetext{
*Corresponding Authors: pdoyle@mit.edu., dirimia@mgh.harvard.edu.

$\perp$ Author Contributions

K.W.B. and J.J.K. contributed equally to this work.

Notes

The authors declare no competing financial interest.

ASSOCIATED CONTENT

Supporting Information

The Supporting Information is available free of charge on the ACS Publications website at DOI: 10.1021/acs.lang-muir.5b03501. Details of the control on incorporated streptavidin concentration and one movie to show the synthesis of the streptavidin-incorporated hydrogel particles using SFL (PDF)
} 


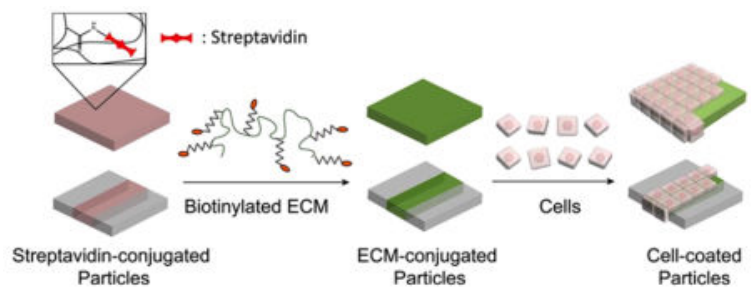

\section{INTRODUCTION}

Multifunctional anisotropic microparticles have been widely used in biomedical applications, such as diagnostics, ${ }^{1-4}$ drug delivery system,${ }^{5}$ cell mimicking, ${ }^{6}$ and tissue engineering. ${ }^{7,8}$ They are commonly synthesized from poly(ethylene glycol) (PEG) and alginate monomers such that they are biocompatibile and their stiffness, porosity, and functionality are highly tunable. As the number of prepolymer solutions available for synthesis increases, the range of applications for these particles is also increasing. Multifunctional microparticles incorporating live cells hold great potential for applications in biotechnology, bioengineering, and biomedical research. For example, microcarrier beads are commonly used for the industrial-scale culture of anchorage-dependent cells and for the production of antibodies, viruses, and stem cell products. ${ }^{9,10}$ Cell-laden microparticles have been utilized as building blocks for the construction of dynamic self-assembled tissues. ${ }^{11-13}$ Cell-adhesive micropallets have been tested for massively parallel clonogenic screening, ${ }^{14}$ single cell sorting, ${ }^{15}$ in vitro therapeutic models, ${ }^{4}$ or the study of cell-microenvironment interaction. ${ }^{7}$ However, for most of these applications, microparticles can accommodate only homogeneous cell cultures and cannot take advantage of the recent advances enabled by cell-patterning technologies. ${ }^{16-20}$

Emerging technologies, such as stop flow lithography (SFL), are well suited to take on the challenge of producing hydrogel microparticles with complex chemical patterns at high throughput. ${ }^{21-25}$ The length scales in SFL are ideally suited for cell culture and engineered cell constructs, for example, by trapping cells in precise positions within the PEG particle during the polymerization steps ${ }^{26}$ However, the PEG particles prepared by SFL are repellent for cell adhesion, and strategies to incorporate cells into the particles expose cells to toxic photoinitiators and monomers, which can trigger phenotypic changes for the encapsulated cells. Moreover, the techniques incorporating cells into particles are not suitable for multifunctional particle synthesis. ${ }^{7,11,12}$ While particle synthesis by ionic cross-linking allows cells to remain intact during particle synthesis, these particles have a homogeneous composition and cell-adhesion properties. ${ }^{5,27,28}$

In this study, we rely on SFL to create anisotropic multifunctional particles that enable cell adhesion on predefined patterns. We attach collagen, the representative extracellular matrix (ECM) materials, and poly-L-lysine (PLL), a cell-adhesion promoter, to the hydrogel particle network by the coupling reaction between amine and $\mathrm{N}$-hydroxysuccinimide (NHS) and streptavidin-biotin conjugation. We allow cells to attach to the collagen/PLL-coated particles. Using this approach, we demonstrate the formation of tightly sealed blood-brainbarrierlike layers of brain endothelial cells on particles. Furthermore, we utilize SFL to 
create heterogeneous cell-laden microparticles by choosing the sequence of EDC coupling and streptavidin-biotin conjugation and pattern breast cancer cells on a narrow strip on these particles.

\section{EXPERIMENTAL SECTION}

\section{Materials}

The PEG monomer solutions consisted of $20 \%$ (v/v) poly(ethylene glycol) (700) diacrylate (PEG-DA 700, Sigma-Aldrich), 40\% (v/v) poly(ethylene glycol) (200) (PEG 200, SigmaAldrich) or PEG (600) (Sigma-Aldrich), 35\% (v/v) 1× phosphate-buffered saline (PBS, Cellgro) with 0.05\% Tween-20 (Sigma-Aldrich) buffer (PBST), and 5\% (v/v) 2-hydroxy-2methylpropiophenone (Sigma-Aldrich). Streptavidin-PEG(2000)-acrylate (SA-PEG-A) was prepared by mixing $10 \mathrm{mg} / \mathrm{mL}$ streptavidin (Invitrogen) in $1 \times$ PBS buffer and succinimidyl carboxy methyl ester (SCM)-PEG (2000)-acrylate (Laysan Bio, Inc.) at a mole ratio of 1:1. SA-PEG-A was mixed into the PEG monomer solutions in a 1:9 (v/v) ratio to give a final concentration of $0.4 \mathrm{mg} / \mathrm{mL}$. All homogeneous particles were made from the prepolymer solutions containing the SA-PEG-A. For chemically anisotropic particle synthesis, prepolymer solution for the cell-adhesive part consisted of 30\% (v/v) PEG-DA (700), 30\% (v/v) acrylic acid (Polysciences), 20\% (v/v) PEG (200), 25\% (v/v) PBST, and 5\% (v/v) 2hydroxy-2-methylpropiophenone. A prepolymer solution of the control side was prepared by substituting acrylic acid with PBST. Biotin-4-fluorescein isothiocyanate (Biotin-4-FITC, Invitrogen) was used to confirm the streptavidin incorporation into the hydrogel particle networks at a concentration of $1 \mathrm{mg} / \mathrm{mL}$ in deionized (DI) water. Biotinylated collagen was prepared by mixing $1 \mathrm{mg} / \mathrm{mL}$ collagen in $0.01 \mathrm{M}$ acetic acid (collagen-FITC, SigmaAldrich) and succinimidyl valerate (SVA)-PEG (3400)-biotin (Laysan Bio, Inc.) in a 50:1 (v/v) ratio and incubating the mixture at $4{ }^{\circ} \mathrm{C}$ overnight. We used SVA because the succinimidyl group in the chemical exhibits a relatively long half-life against hydrolysis. ${ }^{29}$ Biotinlyated poly-L-lysine was purchased (Biotin-PLL-FITC, MW $=25 \mathrm{kDa}$, two to seven biotins conjugated on each PLL molecule, NANOCS). Alternatively, it was prepared by mixing $1 \mathrm{mg} / \mathrm{mL}$ poly-L-lysine (PLL-FITC, MW $=15-30 \mathrm{kDa}$, Sigma-Aldrich) in DI water and SVA-PEG (3400)-biotin. The poly-L-lysine was labeled with fluorescein (FITC) and biotinylated using a similar protocol with that employed for collagen.

\section{Microfluidic Devices}

Microfluidic devices were manufactured by pouring PDMS (Sylgard 184, Dow Corning, mixed at a base-to-curing agent ratio of 10:1) over an SU-8 master and then curing for $2 \mathrm{~h}$ at $60{ }^{\circ} \mathrm{C}$ in an oven. The SU-8 master was made from a negative SU-8 50 photoresist (MicroChem) on a 4 in. silicon wafer to create a mold for particle synthesis channels of 60 $\mu \mathrm{m}$ height. The cured PDMS replica was peeled off of the mold, and inlet holes were punched. The particle synthesis chamber in each device was $1 \mathrm{~cm}$ in length, $300 \mu \mathrm{m}$ in width, and $70 \mu \mathrm{m}$ in height. A reservoir was cut into the PDMS at the end of the chamber to collect the particles. The PDMS devices were cleaned off by sonicating in ethanol for $5 \mathrm{~min}$, rinsing with ethanol, rinsing with water, and drying with argon. Each PDMS device was placed on a partially cured PDMS surface on a glass slide and then sealed by full curing 
overnight at $60{ }^{\circ} \mathrm{C}$ in an oven. The partially cured PDMS surface was prepared by coating PDMS on a glass slide and curing the PDMS for $25 \mathrm{~min}$ at $60{ }^{\circ} \mathrm{C}$.

\section{Particle Synthesis}

For the particle synthesis, the PDMS devices were mounted on an inverted microscope (Axiovert 200, Zeiss) equipped with a VS25 shutter system (UniBlitz) to precisely control the UV exposure dose. Photomasks with an array of in-plane particle shapes were designed using AutoCAD 2012 and printed using a high-resolution printer at Fine Line Imaging (Colorado Springs, CO). The mask was inserted into the field stop of the microscope, and UV light flashed through it using a Lumen 200 (Prior). A filter set that allowed wide UV excitation (11000v2: UV, Chroma) was used to filter out light of undesired wavelengths. SFL was then utilized to synthesize particles using stop, polymerization, and flow times of 500, 200, and $800 \mathrm{~ms}$, respectively. ${ }^{24,30}$ The resulting hydrogel particles were collected in PBST. Tween-20 was required to prevent particle aggregation. Streptavidin incorporated with the particles does not undergo denaturation but maintains its strong affinity for biotin in the Tween-20-containing buffer. ${ }^{3}$ Finally, the particles were rinsed five times with $500 \mu \mathrm{L}$ of PBST and stored at a final concentration of $\sim 10^{4}$ particles $/ \mathrm{mL}$ in a refrigerator $\left(4^{\circ} \mathrm{C}\right)$ for later use.

\section{ECM Conjugation}

Ten microliters of 1 to $2 \mathrm{mg} / \mathrm{mL}$ biotinylated collagen or biotinylated PLL was mixed with $500 \mu \mathrm{L}$ of PBST in an Eppendorf tube. Around 100 particles were introduced into the solution, putting $10 \mu \mathrm{L}$ of the particle solution into the tube. Target incubation was conducted at room temperature for $30 \mathrm{~min}$ in a thermomixer (Multi-Therm, Biomega; used for all incubation steps at a $1500 \mathrm{rpm}$ setting). After the conjugation, particles were washed five times with a rinse solution of $500 \mu \mathrm{L}$ of PBST, using centrifugation at $6000 \mathrm{rpm}$ (Galaxy MiniStar, VWR) to pull particles to the bottom of the tube for manual aspiration and exchange of carrier solution. For all rinses in this study, $50 \mu \mathrm{L}$ of solution was left at the bottom of the tube to ensure the retention of particles. Finally, the collagen or PLL conjugated particles were stored at final concentrations of $\sim 10^{2}$ particles $/ \mathrm{mL}$ in a refrigerator $\left(4{ }^{\circ} \mathrm{C}\right)$ for later use. Collagen and PLL which were not conjugated with biotin were removed during the particle washing steps in ECM conjugation.

\section{Streptavidin Functionalization for Chemically Anisotropic Particles}

Eighty microliters of $20 \mathrm{mg} / \mathrm{mL} N$-hydroxysuccinimide (NHS, Sigma-Aldrich) and $\mathrm{N}$-(3(dimethylamino)propyl)- $N$-ethyl-carbodiimide hydrochloride (EDC, Sigma-Aldrich) in PBST buffer was added to $400 \mu \mathrm{L}$ of PBST with around 100 particles in an Eppendorf tube. The solution was incubated at $21.5^{\circ} \mathrm{C}$ for $30 \mathrm{~min}$, and particles were rinsed with PBST three times. Rinsed particles were stored in $500 \mu \mathrm{L}$ of PBST with $2 \mu \mathrm{g} / \mathrm{mL}$ of neutravidin (Life Technologies), incubated at $21.5^{\circ} \mathrm{C}$ for $2.5 \mathrm{~h}$, and rinsed three times with PBST.

\section{Endothelial Cell Preparation}

Rat brain endothelial cells (RBE4 from INSERM) were cultured in cell culture Petri dishes. ${ }^{31}$ The dishes were coated with collagen type $\mathrm{I}$ at $0.1 \mathrm{mg} / \mathrm{mL}$ by incubating for $5 \mathrm{~min}$ 
at room temperature and rinsing with PBS. Cells were grown in endothelial growth media culturing medium (EGM-2 MV BulletKit: CC-3156 and CC-4147, Lonza Walkersville) supplemented with $1 \%$ penicillin-streptomycin (P/S). To load endothelial cells on particles, gel particles were loaded into 48-well culture plates (CLS3548, Sigma-Aldrich), coated with collagen type I (354249, Becton Dickinson, Franklin Lakes, NJ) at $0.1 \mathrm{mg} / \mathrm{mL}$, and incubated overnight at $37{ }^{\circ} \mathrm{C}$ with $5 \% \mathrm{CO}_{2}$. The cell culture medium was replaced with fresh medium the next day.

\section{Breast Cancer Cell Preparation}

MDA-MB-231 (ATCC) breast cancer cells were cultured in Dulbecco's Modified Eagle's Medium (DMEM, Gibco) with 10\% fetal bovine serum (Invitrogen) and $1 \% \mathrm{P} / \mathrm{S}$ at $37{ }^{\circ} \mathrm{C}$ and $5 \% \mathrm{CO}_{2}$. Cells were split and reseeded at a 1:5 ratio with the medium changed every 3 days. For cell assays, the cells were then removed from the incubator and washed twice with $1 \times$ PBS. After that, cells were detached from the surface using $0.05 \%$ trypsin for $5 \mathrm{~min}$ at $37{ }^{\circ} \mathrm{C}$ and $5 \% \mathrm{CO}_{2}$, quenched with serum-containing culture medium, and centrifuged at $1000 \mathrm{rpm}$ for $5 \mathrm{~min}$. Finally, the cells were resuspended in $1 \mathrm{~mL}$ of culture medium to yield a working concentration of $\sim 10^{6}$ cells $/ \mathrm{mL}$. For the cell assay experiments, $10 \mu \mathrm{L}$ of the cell suspension was pipetted into PDMS reservoirs containing $100 \mu \mathrm{L}$ of the particle solution.

\section{Cell Adhesion}

Particles were added to a reservoir coated with PEG, which covered the glass bottom and PDMS walls. PEG coating was achieved by the controlled spreading of a prepolymer solution of chemically anisotropic particles on the glass, followed by curing. Subsequently, MDA-MB-231 breast cancer cells in a solution medium were the cancer cells on top of particles. After $2 \mathrm{~h}$ of incubation at $37^{\circ} \mathrm{C}$, cell adhesion behavior was observed on the basis of their morphology change. Particles and cells were resuspended by pipetting. Particles settled down quickly because of higher density compared to that of cells. Unattached cells were removed by the aspiration of the solution on top of the particles.

\section{Immunostaining}

We rinsed cells in medium with PBS twice and fixed them by incubating in fresh $4 \%$ paraformaldehyde aqueous solution (157-4, Electron Microscopy Sciences, Washington, PA) for more than $15 \mathrm{~min}$ at room temperature. After two PBS rinses, we permeabilized the cells by incubating in $0.1 \%$ Triton X-100 in PBST (phosphate-buffered saline with $0.1 \%$ Tween-20) for $15 \mathrm{~min}$ at room temperature. To block the nonspecific binding of antibodies, we incubated the cells in $3 \%$ human serum albumin overnight in PBST at $4{ }^{\circ} \mathrm{C}$. We stained cells by incubating with the first antibody for ZO-1 (339100, ZO-1, mouse monoclonal antibody, Invitrogen Corp., Camarillo, CA) at $5 \mu \mathrm{g} / \mathrm{mL}^{-1}$ PBST overnight at $4{ }^{\circ} \mathrm{C}$ and then with the second antibody for ZO-1 (715-586-151, Alexa Fluor 594-conjugated donkey antilmouse IgG, Jackson Immuno Research Laboratory, West Grove, PA) at $1.5 \mu \mathrm{g} / \mathrm{mL}^{-1}$ in PBST for $3 \mathrm{~h}$ at $4{ }^{\circ} \mathrm{C}$ in a dark room. We rinsed solutions with PBST twice after each step from permeabilization to second antibody incubation. We stained the nucleus with mounting oil including a DAPI (17985-51, Fluoro-Gel II, Electron Microscopy Sciences) for $15 \mathrm{~min}$ at room temperature in a dark room before imaging. The covered area was measured with 
ImageJ, cell image analysis software, which automatically identified the cellular boundary by the fluorescence intensity of the cell membrane.

\section{RESULTS AND DISCUSSION Cell Culture on Homogenous Microparticles}

Collagen or PLL-coated PEG particles were generated by the coupling reaction and the interaction of streptavidin and biotin. Acrylate functional groups added to streptavidin and amide bonds were formed between streptavidin and PEG-acrylate via the amine-NHS coupling reaction. The resulting SA-PEG-acrylate was then mixed with PEG monomer solution, thus allowing us to incorporate streptavidin into the polymerization step of SFL (Figure 1A). The precursor solution was then polymerized into a PDMS microfluidic channel under stopped-flow conditions ${ }^{30}$ via UV exposure (Supporting Information Movie $\mathrm{S} 1)$. In this polymerization step, the particle geometry was determined by the mask shape and channel height (Figure 1B). Short UV exposure ( $0.2 \mathrm{~s})$ is sufficient to covalently bond SA-PEG-acrylate to selected volumes of the PEG network during the particle polymerization process. Note that the short duration of UV exposure is compatible with the retention of streptavidin activity. During the "flow" step, the polymerized particles are advected within the surrounding unpolymerized precursor solution and harvested in the collection reservoir.

We validated the incorporation of streptavidin by incubating synthesized particles with biotin-labeled fluorescein isothiocyanate (FITC). Because of the strong interaction between streptavidin and biotin, microparticles exhibited a fluorescence signal after the rinsing of free biotin-FITC (Figure 1C). Moreover, microparticles synthesized from juxtaposed streams of increasing concentrations of streptavidin exhibited a stepwise increase in fluorescence signal intensity that corresponds to increased streptavidin concentration to a given region (Supporting Information Figure S1A). In separate experiments we verified that a simple linear relation exists between the fluorescence signal intensity and the streptavidin concentration (Supporting Information Figure S1B).

Biotinylated collagen or poly-L-lysine (PLL) was added following a similar procedure and was bound selectively on surfaces where streptavidin was present. Collagen was selected as a model ECM to mediate cell attachment to the surface of the particles due to its vast abundance in nature. ${ }^{32}$ Poly-L-lysine is a commercially available synthetic polymer that is positively charged in water and widely used for coating cell culture surfaces to improve cell adhesion by altering the surface charges. Also, PLL promotes neural attachment and is commonly used to culture neurons in an in vitro environment. ${ }^{33}$

Biotinylated collagen or PLL, prepared by reacting with NHS-PEG-biotin, was mixed in ratios determined by the stoichiometry between the mole numbers of amines in collagen or PLL and NHS-PEG-biotin. To confirm the uniform coating of collagen and PLL, we employed FITC-labeled molecules. Streptavidin-incorporated hexagonal particles are homogeneously conjugated with collagen via the streptavidin-biotin interaction (Figure 2A,B). The hexagonal shapes can be useful for close-packing assembly. ${ }^{34}$ In the absence of streptavidin, no collagen was not physically adsorbed to the surface of the particles, as 
supported by the lack of fluorescence of the particles after passage through the fluorescent collagen solution (Supporting Information Figure S2). The results suggest that collagen is covalently conjugated to the particles by the streptavidin-biotin interaction. The homogeneity of the collagen coating suggests that no aggregation of collagen takes place during the procedure. The same strategy could be applied to particles of various shapes; for example, we prepare collagen/PLL-conjugated tubular particles with a high aspect ratio (Figure 2C,D). Such particles are easily toppled over and can be used for end-to-end assembly at fluid interfaces. ${ }^{35}$ These results show that SFL can be used as a general way to attach various ECMs to anisotropic particles based on streptavidin-biotin interaction.

To observe the cell adhesion behavior, MDA-MB-231 breast cancer cells were attached to the collagen-coated particles. After $2 \mathrm{~h}$ of incubation, most of the cells were attached on top of particles and formed spindlelike morphology. Whereas many cells in contact with the glass substrate remain rounded, it is likely that MDA-MB-231 cells have a stronger affinity for collagen-coated particles relative to the surrounding glass substrate (Figure 2E).

\section{Endothelial Cells Culture on Cell-Adhesive Particles}

To further validate the utility of the technology, we characterized the formation of monolayers of rat brain endothelial cells (RBE4) onto collagen I-coated microparticles (Figure 3A-D). The size of the square particles ( $200 \mu \mathrm{m}$ width and $60 \mu \mathrm{m}$ height) is sufficient for the attachment of cells both to the top and sides of the microparticles. For the characterization of the number of RBE4 cells loaded onto the microparticles, we defined a "loading coverage" coefficient, $\gamma_{\mathrm{L}}$, as the ratio between the estimated top surface area of the particle covered by loaded endothelial cells in a monolayer and the top surface area of the original particle. The area was estimated by counting the total cross-sectional area of uniformly loaded cells. The cell coverage of the microparticles increased with the loading cell density (Figure 3D) and exceeded $100 \%$ at the loading cell density of $200 \%$, implying that overloaded RBE4 cells were arranged in multiple layers. The actual cell coverage decreased above the $500 \%$ of the loading cell density, when overgrown RBE4 cells detach from the particle. These results emphasize the importance of precise control over the cell loading for effective cellular coverage of the particles. To evaluate the phenotype of endothelial cells after adhesion to the particles, we also imaged the expression of ZO-1 protein, one of the tight junctions involving proteins between endothelial cells. We found that $\mathrm{ZO}-1$ is robustly expressed at the interface between cells on microparticles.

\section{Spatially Controlled Cell Adhesion on Heterogeneous Particles}

Although we could successfully synthesize five-striped particles that contain different concentrations of streptavidin in each stripe (Supporting Information Figure S1A), this procedure was not suitable for synthesizing heterogeneous particles that have perfect control and a cell-adhesive region. As particles were flushed with unreacted prepolymer solution during synthesis, unreacted streptavidin also bound to the control side because of its high concentration.

To avoid this problem, we switched the sequence of the coupling reaction and streptavidinbiotin conjugation (Figure 4A). Acrylic acid was mixed in a prepolymer solution of a cell- 
adhesive region to provide a carboxylic acid group. This prepolymer solution flowed with the control precursor solution in parallel streams (Figure 4A). The channel Reynolds number in our working regime is $\sim 10^{-3}$, which is sufficiently low to create stable laminar flows. The monomer residence time prior to polymerization is $\sim 0.6 \mathrm{~s}$, which is short enough to prevent diffusion between the interfaces of adjacent flow streams. After particle synthesis, particles were incubated to functionalize neutravidin (a neutrally charged molecule similar to streptavidin). During incubation, carboxylic acid groups on the cell-adhesive side were activated and functionalized by neutavidin via the EDC coupling reaction. This processes required neutravidin concentrations that are 100-fold lower than those used previously, thus avoiding nonspecific binding.

Neutravidin-conjugated multistriped particles were incubated with biotin-conjugated PLL. Using fluorescence imaging, we verified that PLL was patterned only in the particle region containing carboxylic acid (Figure 4B). This result indicates that the neutravidin-biotin interaction and PEG antifouling properties can be used synergistically to pattern cellattractive materials onto polymerized structures with a high degree of spatial control. In this PLL functionalization process, the positive charge of PLL might enhance the specific adhesion due to the possibility of the existence of unreacted carboxylic acid groups, which have negative charges.

The patterning of cells on heterogeneous particles was accomplished in a two-step procedure. First, MDA-MB-231 breast cancer cells were loaded onto PLL-coated heterogeneous particles placed in a nonadhesive reservoir, coated on the bottom and sides with a layer of polymerized PEG. After $2 \mathrm{~h}$ of incubation, the medium inside the reservoir was gently agitated by pipetting, and both particles and nonadherent cells were lifted from the bottom of the well. When allowed to settle, particles sedimented faster than cells due to their larger density. Free-floating cells were removed by carefully extracting the top portion of the medium. This protocol, illustrated in the sequence of images in Figure 4C, is gentle and effective and can be performed as soon as $2 \mathrm{~h}$ after loading the cells. One challenge to be addressed in future work is the relatively low yield of loading cells on particles in the first step of the protocol. The particles thickness and consequent height difference between the top of the particle and the bottom of the well limits the number of cells that settle on top of the particles. It is important to note, however, that the majority of the cells that settled on the cell adhesive pattern of the particles remain attached after the pipetting steps. These cells grow on top of the particles, and their spreading is limited to the pattern (Figure $4 \mathrm{C}$, right panel). Moreover, the patterning process described here for PLL patterning on relatively simple heterogeneous particles can be applied to more complex and multidimensional patterned microparticles. The synthesis of such microparticles by advanced flow lithography techniques such as lock release lithography, ${ }^{23}$ hydrodynamic focusing lithography, ${ }^{24}$ and oxygen-free flow lithography ${ }^{25}$ may produce microparticles with additional chemical and physical properties required for various applications.

\section{CONCLUSIONS}

We developed a versatile method based on SFL, a coupling reaction, and the streptavidinbiotin interaction to create particles with patterns of cell-adhesive surfaces. Compared to 
previous studies on cell-laden particle fabrication, our process offers three important advantages. First, with multiple laminar coflows in a microfluidic device, streptavidinpatterned particles can be conjugated with ECM materials for cell loading and attachment. Our process can be used to significantly expand the limits of chemically and geometrically complex cell-laden particles. Second, our approach also offers tremendous flexibility for tuning the cell affinity in particles by alternating not only collagen and PLL but also any ECM materials. Because most ECM materials have amine groups that can be functionalized with biotin using an amine-NHS coupling reaction, our approach can be used to incorporate virtually any kind of ECM material with amine groups into anisotropic multifunctional particles. Finally, when cells are loaded onto particles after the completion of UV polymerization, this method can minimize physiological changes to the cells. The photopolymerization process requires UV light, a photo-initiator, and monomers, each of which negatively affects the physiology of cells. We believe that our approach can ultimately be useful for the mass production of new classes of cell-laden particles as building blocks for tissue-engineered constructs.

\section{Supplementary Material}

Refer to Web version on PubMed Central for supplementary material.

\section{Acknowledgments}

We gratefully acknowledge support from the National Institutes of Health (GM092804), the National Science Foundation (CMMI-1120724 and DMR-1006147), and a Samsung Scholarship to J.J.K. We also thank Lynna Chen and Rathi Srinivas for insightful discussions.

\section{References}

1. Pregibon DC, Toner M, Doyle PS. Multifunctional encoded particles for high-throughput biomolecule analysis. Science. 2007; 315(5817):1393-1396. [PubMed: 17347435]

2. Appleyard DC, Chapin SC, Doyle PS. Multiplexed protein quantification with barcoded hydrogel microparticles. Anal Chem. 2011; 83(1):193-9. [PubMed: 21142122]

3. Chapin SC, Appleyard DC, Pregibon DC, Doyle PS. Rapid microRNA profiling on encoded gel microparticles. Angew Chem, Int Ed. 2011; 50(10):2289-93.

4. Chung SE, Kim J, Yoon OD, Song Y, Hoon Lee S, Min S, Kwon S. One-step pipetting and assembly of encoded chemical-laden microparticles for high-throughput multiplexed bioassays. Nat Commun. 2014; 5:3468. [PubMed: 24632848]

5. Hu Y, Wang Q, Wang J, Zhu J, Wang H, Yang Y. Shape controllable microgel particles prepared by microfluidic combining external ionic crosslinking. Biomicrofluidics. 2012; 6(2):026502.

6. Haghgooie R, Toner M, Doyle PS. Squishy Non-Spherical Hydrogel Microparticles. Macromol Rapid Commun. 2010; 31:128-134. [PubMed: 21590884]

7. Eng G, Lee BW, Parsa H, Chin CD, Schneider J, Linkov G, Sia SK, Vunjak-Novakovic G. Assembly of complex cell microenvironments using geometrically docked hydrogel shapes. Proc Natl Acad Sci U S A. 2013; 110(12):4551-4556. [PubMed: 23487790]

8. Chung SE, Park W, Shin S, Lee SA, Kwon S. Guided and fluidic self-assembly of microstructures using railed microfluidic channels. Nat Mater. 2008; 7(7):581-7. [PubMed: 18552850]

9. van Hemert P, Kilburn DG, van Wezel AL. Homogeneous cultivation of animal cells for the production of virus and virus products. Biotechnol Bioeng. 1969; 11(5):875-85. [PubMed: 5361174] 
10. Wang Y, Cheng L, Gerecht S. Efficient and scalable expansion of human pluripotent stem cells under clinically compliant settings: a view in 2013. Ann Biomed Eng. 2014; 42(7):1357-72. [PubMed: 24132657]

11. Du YA, Ghodousi M, Lo E, Vidula MK, Emiroglu O, Khademhosseini A. Surface-Directed Assembly of Cell-Laden Microgels. Biotechnol Bioeng. 2010; 105(3):655-662. [PubMed: 19777588]

12. Koh WG, Itle LJ, Pishko MV. Molding of hydrogel multiphenotype cell microstructures to create microarrays. Anal Chem. 2003; 75(21):5783-5789. [PubMed: 14588018]

13. Du Y, Lo E, Ali S, Khademhosseini A. Directed assembly of cell-laden microgels for fabrication of 3D tissue constructs. Proc Natl Acad Sci U S A. 2008; 105(28):9522-7. [PubMed: 18599452]

14. Shah PK, Walker MP, Sims CE, Major MB, Allbritton NL. Dynamics and evolution of betacatenin-dependent Wnt signaling revealed through massively parallel clonogenic screening. Integr Biol (Camb). 2014; 6(7):673-84. [PubMed: 24871928]

15. Shah PK, Herrera-Loeza SG, Sims CE, Yeh JJ, Allbritton NL. Small sample sorting of primary adherent cells by automated micropallet imaging and release. Cytometry, Part A. 2014; 85(7):6429.

16. Dermutz H, Gruter RR, Truong AM, Demko L, Voros J, Zambelli T. Local polymer replacement for neuron patterning and in situ neurite guidance. Langmuir. 2014; 30(23):7037-46. [PubMed: 24850409]

17. Wendeln C, Ravoo BJ. Surface patterning by microcontact chemistry. Langmuir. 2012; 28(13): 5527-38. [PubMed: 22263907]

18. Yan J, Sun Y, Zhu H, Marcu L, Revzin A. Enzyme-containing hydrogel micropatterns serving a dual purpose of cell sequestration and metabolite detection. Biosens Bioelectron. 2009; 24(8): 2604-10. [PubMed: 19251408]

19. Lee JY, Revzin A. Merging photolithography and robotic protein printing to create cellular microarrays. Methods Mol Biol. 2011; 671:195-206. [PubMed: 20967631]

20. Folch A, Toner M. Microengineering of cellular interactions. Annu Rev Biomed Eng. 2000; 2:22756. [PubMed: 11701512]

21. Dendukuri D, Pregibon DC, Collins J, Hatton TA, Doyle PS. Continuous-flow lithography for high-throughput microparticle synthesis. Nat Mater. 2006; 5(5):365-9. [PubMed: 16604080]

22. Dendukuri D, Gu SS, Pregibon DC, Hatton TA, Doyle PS. Stop-flow lithography in a microfluidic device. Lab Chip. 2007; 7(7):818-828. [PubMed: 17593999]

23. Bong KW, Pregibon DC, Doyle PS. Lock release lithography for 3D and composite microparticles. Lab Chip. 2009; 9(7):863-6. [PubMed: 19294294]

24. Bong KW, Bong KT, Pregibon DC, Doyle PS. Hydrodynamic focusing lithography. Angew Chem, Int Ed. 2010; 49(1):87-90.

25. Bong KW, Xu J, Kim JH, Chapin SC, Strano MS, Gleason KK, Doyle PS. Nonpolydimethylsiloxane devices for oxygen-free flow lithography. Nat Commun. 2012; 3:805. [PubMed: 22549834]

26. Panda P, Ali S, Lo E, Chung BG, Hatton TA, Khademhosseini A, Doyle PS. Stop-flow lithography to generate cell-laden microgel particles. Lab Chip. 2008; 8(7):1056-1061. [PubMed: 18584079]

27. Tan WH, Takeuchi S. Monodisperse Alginate Hydrogel Microbeads for Cell Encapsulation. Adv Mater. 2007; 19(18):2696-2701.

28. Maeda K, Onoe H, Takinoue M, Takeuchi S. Controlled Synthesis of 3D Multi-Compartmental Particles with Centrifuge-Based Microdroplet Formation from a Multi-Barrelled Capillary. Adv Mater. 2012; 24(10):1340-1346. [PubMed: 22311473]

29.

http://www.laysanbio.com/clientuploads/Hydrolysis_Half_Lives.pdf.

30. Bong KW, Chapin SC, Pregibon DC, Baah D, Floyd-Smith TM, Doyle PS. Compressed-air flow control system. Lab Chip. 2011; 11(4):743-7. [PubMed: 21116544]

31. Roux F, Couraud PO. Rat brain endothelial cell lines for the study of blood-brain barrier permeability and transport functions. Cell Mol Neurobiol. 2005; 25(1):41-58. [PubMed: 15962508] 
32. Di Lullo GA, Sweeney SM, Korkko J, Ala-Kokko L, San Antonio JD. Mapping the ligand-binding sites and disease-associated mutations on the most abundant protein in the human, type I collagen. J Biol Chem. 2002; 277(6):4223-31. [PubMed: 11704682]

33. Taylor AM, Blurton-Jones M, Rhee SW, Cribbs DH, Cotman CW, Jeon NL. A microfluidic culture platform for CNS axonal injury, regeneration and transport. Nat Methods. 2005; 2(8):599-605. [PubMed: 16094385]

34. Park W, Han S, Lee H, Kwon S. Free-floating amphiphilic picoliter droplet carriers for multiplexed liquid loading in a microfluidic channel. Microfluid Nanofluid. 2012; 13(3):511-518.

35. Lewandowski EP, Cavallaro M, Botto L, Bernate JC, Garbin V, Stebe KJ. Orientation and SelfAssembly of Cylindrical Particles by Anisotropic Capillary Interactions. Langmuir. 2010; 26(19): 15142-15154. [PubMed: 20806978] 


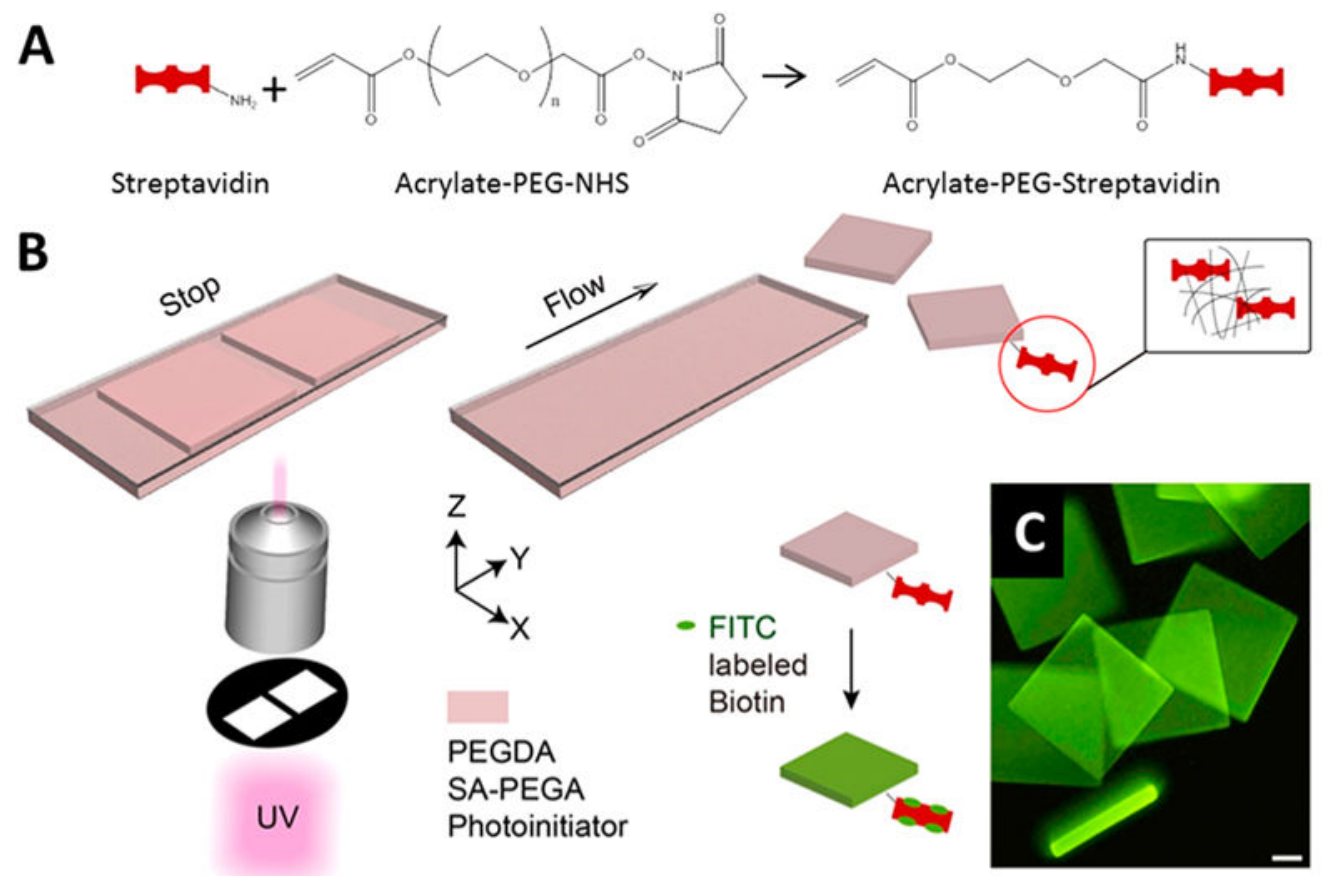

Figure 1.

Synthesis of streptavidin-incorporated particles using SFL. (A) Streptavidin was functionalized with the acrylate group via the amine-NHS coupling reaction. The acrylate group provides the covalent bonding between streptavidin and the PEG network of particles. (B) Particles are photo-cross-linked within a PDMS microchannel by UV light $(365 \mathrm{~nm})$ through a photomask and a $20 \times$ objective lens. During UV polymerization, the acrylatefunctionalized streptavidin is conjugated with the poly(ethylene glycol) (PEG) monomers, which effectively integrates streptavidin into the hydrogel particle networks. Particles are squares of $200 \mu \mathrm{m}$ width and $60 \mu \mathrm{m}$ height. (C) The polymerized particles are then functionalized with biotinylated biomolecules (e.g., biotin-fluorescein isothiocyanate (biotin-FITC)) via the interaction of streptavidin and biotin. All scale bars are $50 \mu \mathrm{m}$. 


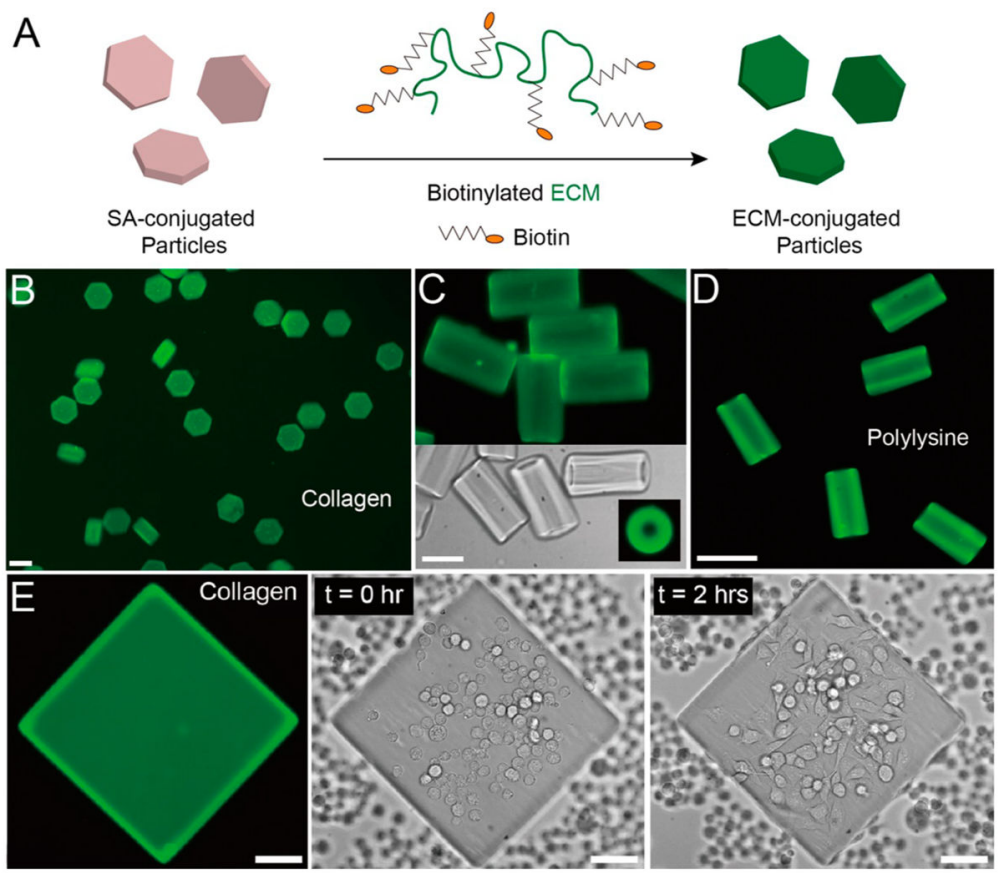

Figure 2.

Cell-attractive material coating and cell-adhesive experiment with homogeneous particles. (A) Schematic description to coat biotin-conjugated collagen/PLL to hexagonal particles via the streptavidin-biotin interaction. (B) Fluorescent image of collagen-conjugated hexagonal particles. (C, D) The streptavidin-incorporated microtubular particles are created by simply changing the photomask feature during the flow lithography process. These particles are then incubated with biotinlyated ECM materials such as collagen and poly-L-lysine. On the basis of the fluorescence signal intensity, these particles are homogeneously conjugated with ECM materials. (E) Cell adhesion on collagen coated particles. Fluorescein-labeled collagen was grafted with square particles prepared by SFL (left). MDA 231 breast cancer cells settled down on top of the particles (middle). After $2 \mathrm{~h}$ of incubation, the cells were attached to the particles, exhibiting the characteristic spindlelike morphology (right). Scale bars are $100 \mu \mathrm{m}(\mathrm{B}), 30 \mu \mathrm{m}$ (C), and $50 \mu \mathrm{m}$ (D and E). 

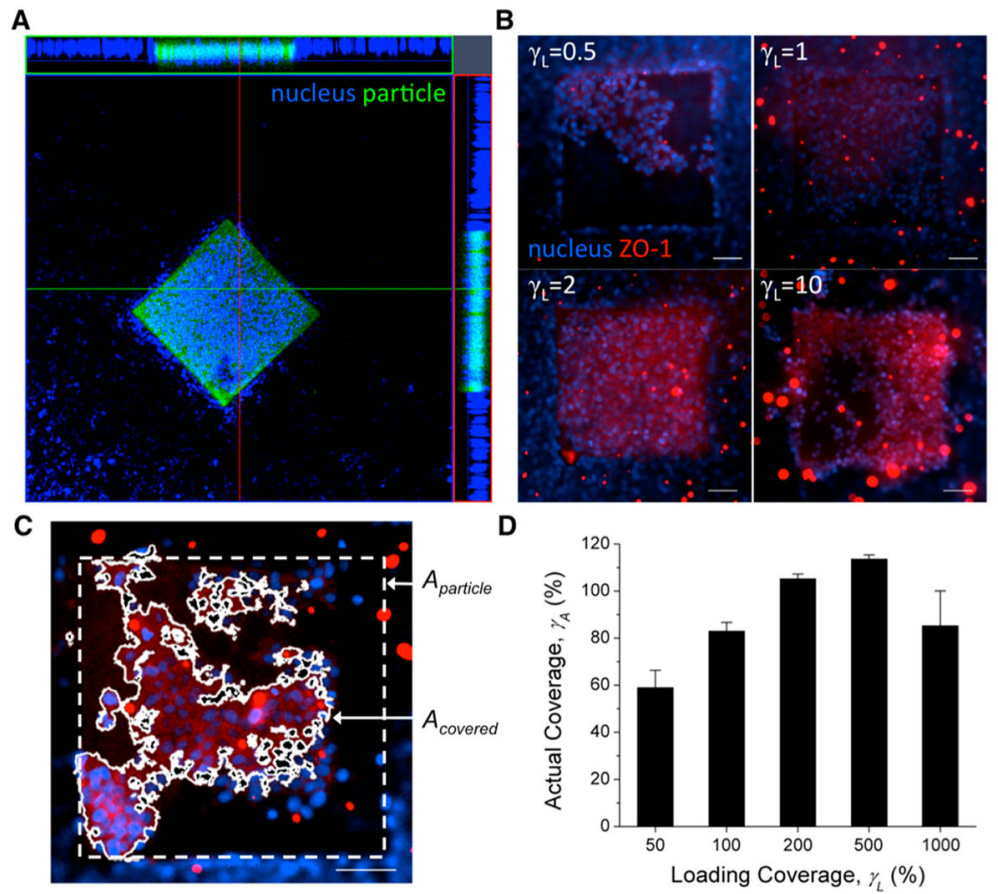

Figure 3.

Rat brain endothelial cells (RBE4) on collagen-conjugated square particles. (A) An FITClabeled particle is covered with RBE4 on all sides. The nucleus of the RBE4 cells is stained in blue using a DNA binding dye. (B) The formation of monolayers and the tightness are validated by imaging a nucleus and a tight junction involving protein, $\mathrm{ZO}-1$, respectively, with the variation of loading coverage, $\gamma_{\mathrm{L}}\left(=A_{\text {cell }} / A_{\text {particle }}\right)$. The bright circular spots on images represent dye particles that were not removed by gentle washing steps compatible with these gel particles. These spots were excluded from quantification by adjusting the upper threshold of fluorescence intensity. (C) Cellular attachment is characterized by an actual coverage, $\gamma_{\mathrm{A}}\left(=A_{\text {covered }} / A_{\text {particle }}\right)$ after 1 day of culturing on the particles. (D) The cellular attachment increases with the loading coverage, but overloading causes cellular aggregation rather than attachment and peeling-off from particles. All scale bars are $50 \mu \mathrm{m}$. 


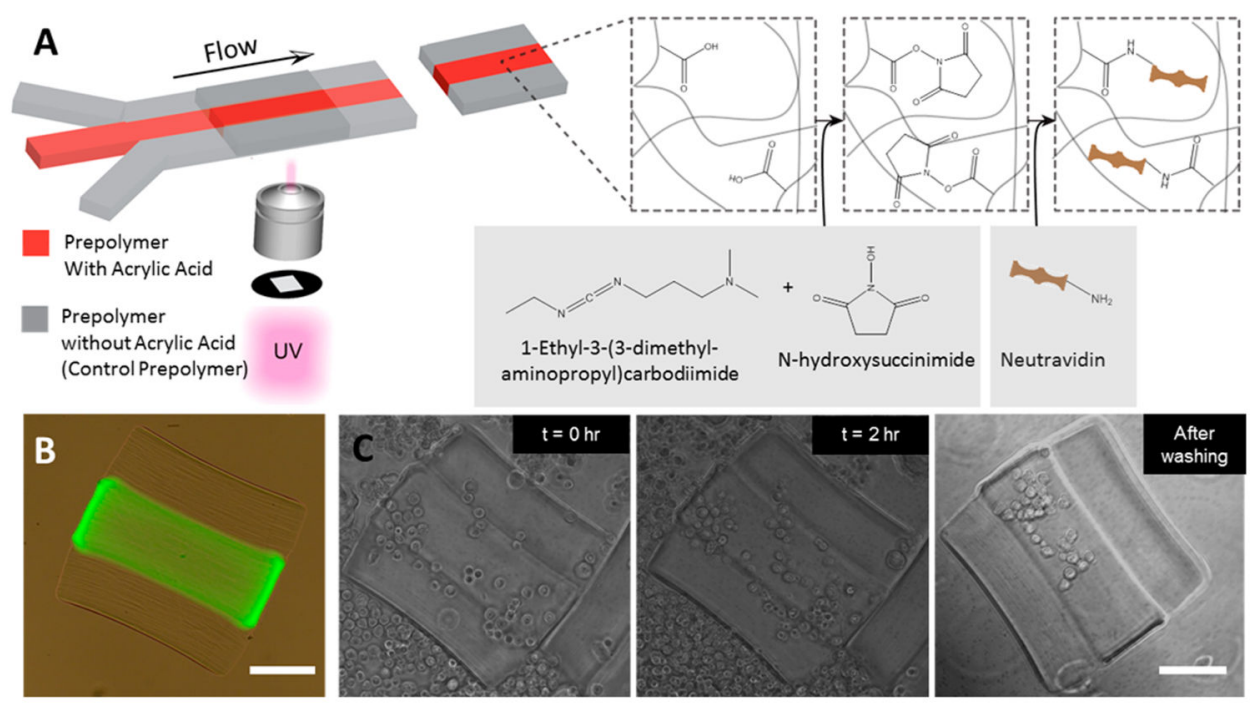

Figure 4.

Spatial cell coating on anisotropic particles. (A) Schematics for anisotropic particle synthesis by SFL and specific neutravidin functionalization via the carbodiimide coupling reaction. (B) Bright field (left) and fluorescent images (right) of anisotropic particles after PLL coating were overlapped to show the specific PLL coating in the middle region. Only the middle region, which contains the carboxylic acid group initially, was selectively coated by PLL via the neutravidin-biotin interaction. (C) Spatial cell attachment on PLL-coated anisotropic particles. MDA 231 breast cancer cells were placed and incubated on the particle surface for $0 \mathrm{~h}$ (left) and $2 \mathrm{~h}$ (middle). After $2 \mathrm{~h}$ of incubation, the solution was agitated, and MDA-MB-231 adhered only in the middle region of particles (right). Left and middle images represent the same particles. Scale bars are $100 \mu \mathrm{m}$. 